21 Law, L. W., and Chang, S. A., Proc. Soc. Exp. Biol. Med., 163, 420 (1971).

22 Duc-Nguyen, H., J. Virol., 2, 643 (1968).

\section{A Critique of McClare's Quantum Mechanical Muscle Model}

THE explanation of muscle contraction by $\mathrm{McClare}^{1,2}$ is at variance with the current tenets of molecular spectroscopy. Lifetime measurements have shown that, in most cases, the excimer has a shorter half-life than that of the parent monomer ${ }^{3}$. In pyrene, the example quoted by McClare, the half-life (at room temperature) of the monomer is $31 \times 10^{-8} \mathrm{~s}$, while that of the excimer is only $4.4 \times 10^{-8} \mathrm{~s}$.

Azumi and McGlynn ${ }^{4}$ observed an excimer emission from solid solutions of pyrene with a lifetime of $10^{-2} \mathrm{~s}$, which they attributed to "an inherent forbidden character of excimer fluorescence itself". It was later found ${ }^{5}$ that their delayed fluorescence arose from an excimer produced by triplet-triplet annihilation; that is, the emission owed its longevity to the delay in the production of the emitting species and not to its slow decay.

I have shown ${ }^{6}$ that two molecules in the lowest triplet state will not interact to yield an excited singlet species (monomer or excimer) if they are situated $3.0 \mathrm{~nm}$ apart. Thus McClare's oscillators, even if they are postulated to be in the first excited triplet state, would not interact and come closer. If the initial distance is changed to $1.0 \mathrm{~nm}$, electron exchange interaction leading first to an excited singlet monomer and later to an excimer will occur; but then the excimer will not be long-lived.

If the excimer has a centre of symmetry, excimer fluorescence will be dipole-forbidden 7 . Experimental evidence, however, suggests that random thermal motion of the two monomers can lower the symmetry and make the transition dipoleallowed. In a rigid matrix, a symmetrical excimer, once formed, will be unable to radiate; but in such a matrix the two oscillators will be prevented from coming closer and McClare's model would fail.

McClare also presents the analogy of the lowest triplet state of chrysene which can be stabilized for as long as a second, and argues that an excimer could also be similarly stabilized. Although the radiative transition of a symmetrical excimer is dipole-forbidden, as is the phosphorescence from the triplet state of an organic molecule, there is an essential difference between the two species. The first triplet state is the lowest excited state of the moleculc, but the singlet excimer state is not; the excimer can always decay by crossing over to its (lower lying) triplet state. Hence a symmetrical excimer cannot be stabilized for as long as the lowest triplet state of an organic molecule.

Department of Chemistry,

K. RAZI NAQVI

The University, Sheffield $S 37 \mathrm{HF}$

Received November 20, 1972; revised February 2, 1973.

1 McClare, C. W. F., Nature, 240, 88 (1972).

2 McClare, C. W. F., J. Theor. Biol., 35, 569 (1972).

3 Birks, J. B., in Photophysics of Aromatic Molecules, 351 (John Wiley, New York, 1970).

${ }^{4}$ Azumi, T., and McGlynn, S. P., J. Chem. Phys., 39, 3533 (1963).

5 Smith, F. J., and McGlynn, S. P., J. Chem. Phys., 42, 4308 (1965).

6 Razi Naqvi, K., Chem. Phys. Lett., 1, 497 (1967).

7 Hoijtink, G. J., Z. Elektrochem., 64, 156 (1960).

\section{McClare's Quantum Mechanical Muscle Model}

STARTING from the belief that the "energy" for muscular contraction is derived from the "energy" released by the hydrolysis of ATP, McClare ${ }^{1}$ has concluded that this "energy" must initially be stored in individual molecules of ATP. The amount of "energy" so stored is supposed to equal the enthalpy change for the hydrolysis of ATP (presumably in its ground state), at $p \mathrm{H} \mathrm{7.4.} \mathrm{A} \mathrm{considerable} \mathrm{part} \mathrm{of} \mathrm{the} \mathrm{quoted} \mathrm{figure}$ $\left(-40 \mathrm{~kJ} \mathrm{~mol}^{-1}\right)$ is, in fact, due to the heat of neutralization of a proton which is formed in near stoichiometric amount at this $p \mathrm{H}$ (refs. 2, 3). Nevertheless, McClare proposes that the total enthalpy change is somehow trapped in the other two products of hydrolysis, ADP and inorganic phosphate, which subsequently exist in an excited "state", out of thermal equilibrium with surrounding molecules for a significant time. A calculation then shows that the wavelength of radiation having energy equivalent to this enthalpy change is $3 \mu \mathrm{m}$, which lies in the infrared. McClare concludes from this that the "energy" is stored (in ADP and inorganic phosphate) in a vibrational mode. The lifetime of a vibrational excited state is quoted as being $c a \cdot 10^{-7} \mathrm{~s}$.

But this figure is typical only for dilute gases of small molecules ( 2 to 6 atom at $10^{-2}-10^{-3} \mathrm{~atm}$ ) where the lifetime is collisionally limited. At much lower pressures (for example, in outer space), the lifetime of a single vibrationally excited molecule is its radiative lifetime, which for ordinary molecules is of the order of $10^{-4} \mathrm{~s}$. Under such conditions, the excited energy-level is sharp, with a linewidth of about $5 \times 10^{-8} \mathrm{~cm}^{-1}$. In aqueous media, on the other hand, vibrational energy-levels as revealed by infrared absorption bands are rather broad (typically $\Delta \bar{v}=100 \mathrm{~cm}^{-1}$ ) and the lifetimes of the vibrational excited "states" are correspondingly smaller, by perhaps about 10 orders of magnitude. This may be simply seen as follows. In a condensed phase, initially formed vibrational excited "states" are coupled intra- and intermolecularly (by "resonant transfer" in McClare's terminology) to a quasi-continuum of other vibrational "states". A quantum-mechanical treatment of uniform coupling ${ }^{4-7}$ gives the first order decay lifetime of an initially excited zeroth-order "state" as $\tau=5.3 \times 10^{-12} \times$ $(\Delta \bar{v})^{-1} \mathrm{~s}(\tau \times \Delta \bar{v}=\hbar / h c)$. With $\Delta \bar{\nabla}=100 \mathrm{~cm}^{-1}, \tau=5 \times 10^{-14} \mathrm{~s}$; that is, 6 or 7 orders of magnitude less than the figure given by McClare. Another indication of such short vibrational lifetimes comes from, for example, the feasibility of dye lasers which otherwise would not work.

This means that for all practical purposes vibrational energies of molecules in aqueous solution are accurately distributed according to the Boltzmann Law, that is, they are thermally equilibrated. It is not possible to isolate a vibrational mode from thermal equilibrium for the time required by McClare. Even if the hydrolysis of ATP takes place on the surface of elements of the myofibril system and not in free solution the situation is unlikely to be very different. In any case, the value of the enthalpy-change would then be unknown and might well be close to zero if a proton were not produced in near stoichiometric amount.

McClare is led to "restate" the second law because of his initial premise that ATP can store and provide "energy" while remaining in steady state. The model for muscle contraction which follows requires the isolation of a particular vibrational mode from thermal equilibrium in a condensed phase, and this is not physically possible-unless, of course, Maxwell's Demon intervenes. We note that he has accused a number of muscle physiologists of invoking the assistance of this particular entity.

There can be little doubt that the behaviour of biological systems conforms to the laws of classical thermodynamics as usually understood. There is in principle no particular difficulty in calculating the value of the change in Gibbs function for a muscle contraction providing that the concentrations of all those chemical species which show a net change in amount can be measured before and after contraction. In practice this is obviously technically difficult. But no one has reported a positive value for the change in Gibbs function in these circumstances and until they do we can continue to believe that muscle contraction conforms to the second law.

Were the opening sentence of McClare's article true, that is, if the physiology, biochemistry and structure of muscle were now well understood, there would be no need for models at all. The fact is that the molecular events associated with muscle 